\title{
Incidental findings on magnetic resonance imaging of the internal auditory meatus performed to investigate audiovestibular symptoms
}

\author{
$\mathrm{H} \mathrm{M} \mathrm{HTUN}^{1}$, S L MUI ${ }^{2}$, C WILLIAMS ${ }^{1}$, P S HANS ${ }^{1}$ \\ Departments of ${ }^{1}$ Otolaryngology and ${ }^{2}$ Radiology, Blackpool Victoria Hospital, UK
}

\begin{abstract}
Objective: To determine the frequency of incidental findings found on magnetic resonance imaging scans of the internal auditory meatus performed to investigate audiovestibular symptoms, and to determine how to best manage these when found.

Method: A retrospective review was conducted of internal auditory meatus magnetic resonance imaging scans during a three-month period in the radiology department at a UK district general hospital.

Results: A total of 109 scans were reviewed. Of these, 92.7 per cent showed no retrocochlear pathology, 0.9 per cent showed vestibular schwannoma, 6.4 per cent revealed vascular loops, and 2.8 per cent showed incidental findings that warranted further action and investigation. Of the scans, 40.4 per cent showed other incidental pathologies such as age-related ischaemic changes, and sinus disease that required no further intervention. Of the magnetic resonance imaging scans reviewed, 49.5 per cent were entirely normal.

Conclusion: Almost half of the scans investigating audiovestibular symptoms showed incidental findings. Otolaryngologists should have an understanding of the significance of the most commonly encountered incidental findings, and should counsel patients appropriately and refer them onward when necessary.
\end{abstract}

Key words: MRI; Internal Auditory Meatus; Audiovestibular Symptoms; Vestibular Schwannoma; Incidental Findings

\section{Introduction}

Vestibular schwannomas, or acoustic neuromas, are benign, slow-growing tumours of the vestibular portion of the vestibulocochlear (VIIIth) cranial nerve. ${ }^{1}$ Presentation is variable, with patients most commonly presenting with asymmetrical sensorineural hearing loss (SNHL). ${ }^{2}$ Tinnitus, vertigo, disequilibrium and facial palsy can also be diagnostic indicators, making vestibular schwannoma a challenge to accurately diagnose clinically. ${ }^{2-4}$ Radiological assessment is therefore crucial, with gadolinium-enhanced magnetic resonance imaging (MRI) of the internal auditory meatus recognised as the 'gold standard' for investigating asymmetrical SNHL. 5

With such wide-ranging symptomatology for vestibular schwannoma, increasing numbers of MRI scans of the internal auditory meatus are being performed. ${ }^{6}$ This has led to a rise both in the number of scans reported as normal and those with incidental clinical findings. ${ }^{7}$ There is a growing body of literature addressing the occurrence of incidental findings on
MRI scans performed both in healthy individuals and in those presenting with suspected vestibular schwannoma. ${ }^{8,9}$

This study aimed to determine the frequency of incidental findings on MRI scans of the internal auditory meatus within our department, and to suggest how to best manage these findings when identified.

\section{Materials and methods}

We performed a retrospective review of all acoustic protocol MRI scans performed over a three-month period between May and July 2015 at the Radiology Department of Blackpool Victoria Hospital. All scans, authorised by one of four consultant otorhinolaryngologists, were performed for: asymmetrical SNHL, persistent unilateral tinnitus or other unexplained audiovestibular symptoms.

The standard protocol for the MRI scans was T2weighted, three-dimensional constructive interference in steady state imaging, with $0.7 \mathrm{~mm}$ sections through 
the internal auditory meatus and $5 \mathrm{~mm}$ sections through the brain.

Reports on all scans were reviewed. Exclusion criteria included surveillance scans for known vestibular schwannoma $(n=8)$ and cases in which a full MRI could not be obtained because of patient factors $(n=1)$. A total of 109 MRI scans were assessed and included in the study.

\section{Results}

Of the 109 scans, 8 were reported to show an abnormal internal auditory meatus: there was 1 small vestibular schwannoma (0.9 per cent) and 7 vascular loops (6.4 per cent) (Figure 1). The indication for the MRI scan that revealed a vestibular schwannoma was unilateral SNHL. The indications for the MRI scans that revealed vascular loops were: unilateral SNHL $(n=2)$, tinnitus $(n=3)$ and vertigo $(n=2)$.

A significant proportion (92.7 per cent; $n=101)$ of the 109 scans were found to have a 'normal' internal auditory meatus; no other findings were reported for 54 of them (49.5 per cent). However, 28 scans (25.7 per cent) demonstrated old ischaemic changes, small vessel disease or degenerative changes that did not warrant any further investigations, except for informing these patients' general practitioners. The age range of patients with these findings was 46-87 years, with a median age of 62 years.

Nine patients ( 8.3 per cent) were found to have paranasal sinus disease and evidence of fluid in the mastoid air cells. Seven patients (6.4 per cent) had non-specific findings that were of little clinical importance, such as cava septum pellucidum and dilated Virchow-Robin space.
The remaining three patients (2.8 per cent) required further action. One patient was found to have a deep lobe parotid tumour that required surgical intervention, another had a possible empty sella with benign intracranial hypertension that warranted referral to neurosurgeons, and the last patient had demyelinating changes and needed to be reviewed by neurologists.

\section{Discussion}

As stated above, MRI scanning is regarded as the gold standard investigation for ruling out internal auditory meatus tumours such as vestibular schwannoma, because of its cost-effectiveness, accuracy and superiority over other audiovestibular investigations such as auditory brainstem response (ABR). ${ }^{10,11}$ Our study has demonstrated, however, that only a small proportion ( 0.9 per cent) of scans requested actually demonstrated retrocochlear pathology. This is in line with other studies, which have shown that none of the various clinical criteria that exist to try to increase the pick-up rate of vestibular schwannoma are particularly effective, with none improving beyond a 4 per cent pick-up rate, ${ }^{1-3,6}$ and an incidental finding is more likely to be reported.

\section{Vascular loops}

Vascular loops were detected in 6.4 per cent of patients in our cohort. This conforms with previous literature, with studies by Papanikolaou et $a l^{7}$ and Gupta et $a l .{ }^{12}$ showing incidence rates of 1 per cent and 8.66 per cent respectively. A systematic review by Chadha et al. showed an association between vascular loops in contact with the VIIIth cranial nerve, and asymmetrical SNHL and pulsatile tinnitus; however,

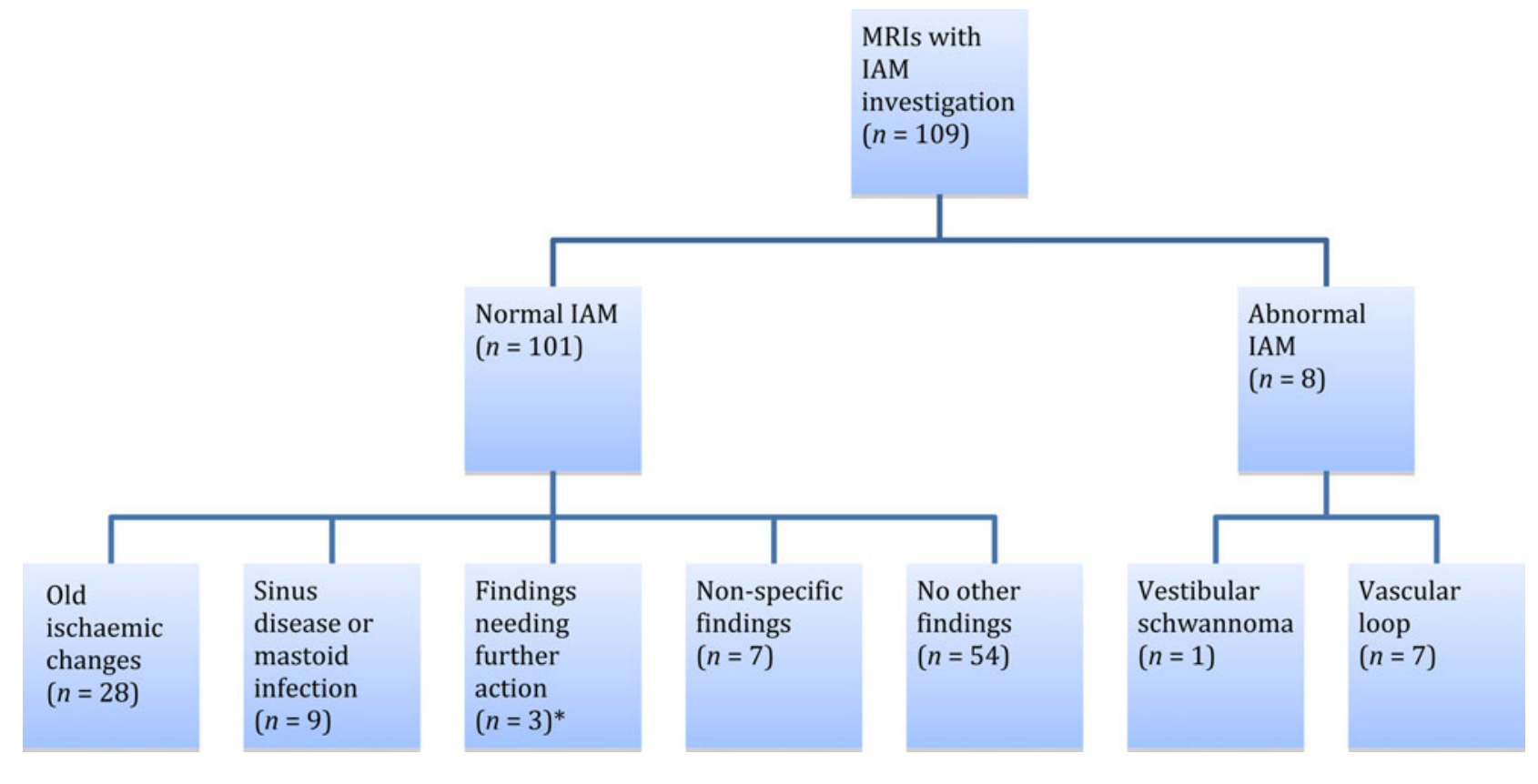

FIG. 1

Various magnetic resonance imaging (MRI) findings in patients with unilateral audiovestibular symptoms. ${ }^{*}$ These were: an empty sella with benign intracranial hypertension, a deep lobe parotid tumour and demyelinating changes. IAM = internal acoustic meatus 
in clinical practice, these are regarded as normal variants and can be present in up to 33 per cent of the population. ${ }^{13}$

If a vascular loop is found to be in contact with the affected VIIIth cranial nerve, with no other causative pathology identified, neurosurgical referral could be considered. Techniques such as decompression of the internal auditory meatus, or insertion of a Silicon or Teflon cushion between the compressing artery and VIIIth cranial nerve, have been described in the literature. ${ }^{14,15}$ Microvascular decompression is not widely undertaken for audiovestibular symptoms because of its invasive nature. None of the patients in our study were disturbed enough by their symptoms to consider neurosurgical intervention.

\section{Ischaemic white matter changes}

The largest proportion of incidental findings in our cohort concerned white matter changes, small vessel disease and previous ischaemic damage (25.7 per cent). Myelin and axonal loss due to small vessel disease or ischaemia is detected as white matter hyperintensities on T2-weighted MRI scans. This is secondary to chronic hypoperfusion of the white matter and disruption of the blood-brain barrier, which leads to chronic leakage of plasma into the white matter. ${ }^{16}$

In the Rotterdam Scan Study, a population-based MRI study, 1077 members of the public underwent MRI. ${ }^{17}$ The findings showed that the prevalence and degree of white matter change increased significantly with age. The age range of patients with these findings in our study was 46-87 years, with a median age of 62 years. Debette and Markus demonstrated an increased risk of stroke and dementia in patients with white matter hyperintensities on MRI scans. ${ }^{18}$ Otorhinolaryngologists should therefore be prepared to screen these patients for stroke and dementia, or, at least, inform the patients' general practitioners so that preventative or disease-modifying measures can be implemented.

\section{Paranasal sinus and mastoid disease}

Of our patients, 8.3 per cent had paranasal sinus or mastoid disease found on MRI scans. The rate of incidental paranasal sinus disease on T2-weighted MRI scans in the literature ranges from 24.7 per cent ${ }^{19}$ to 49.2 per cent. ${ }^{20}$ These variable rates can be influenced by environmental factors such as scanning in the winter, and patient risk factors such as sex, age, smoking and previous sinus disease, which all increase the incidence of incidental sinus disease. ${ }^{21}$

\section{Demyelinating disease}

One of our patients was found to have demyelinating changes and was subsequently referred to the neurology department. In the absence of clinical symptoms and signs suggestive of multiple sclerosis, no further investigations were required.
The term 'radiologically isolated syndrome' was first used by Okuda et al., ${ }^{22}$ in 2009, to describe patients who fulfilled the Barkhof criteria for the diagnosis of multiple sclerosis based on MRI findings, in the absence of any symptoms or clinical findings suggestive of multiple sclerosis. ${ }^{23}$ These authors found that more than 50 per cent of patients with radiologically isolated syndrome showed radiological progression of demyelination, and 30 per cent went on to be diagnosed with multiple sclerosis. ${ }^{22}$ Radiologically isolated syndrome can therefore be considered as a precursor for the development of multiple sclerosis. Onward referral to neurologists is highly recommended for these patients, even though they may be free of clinical signs and symptoms at the time of diagnosis.

\section{Anatomical variants}

We encountered anatomical variants such as dilated Virchow-Robin spaces and cavum septum pellucidum, which are of no clinical relevance. However, to the untrained otorhinolaryngological observer, these findings could cause concern if not flagged by the reporting radiologist as being of no clinical relevance. Other anatomical variants that have little health relevance are mega cisterna magna, callosal lipoma and asymmetrical ventricles. ${ }^{24}$

\section{Cost-effectiveness and alternatives}

The cost-effectiveness of performing MRI scans to diagnose vestibular schwannoma has long been the subject of debate. Limitations of both contrastenhanced computed tomography (CT) and ABR have been discussed, with studies such as that by Raber et al. showing that contrast-enhanced $\mathrm{CT}$ is no more accurate than ABR yet three times more costly. ${ }^{11}$ Furthermore, ABR has low sensitivity and specificity compared to MRI in detecting lesions causing asymmetrical SNHL. ${ }^{25}$ However, cost-analysis concerns should not dictate or discourage the clinical investigation of vestibular schwannoma, as delayed diagnosis can impact on the preservation of the facial nerve and auditory function during any subsequent surgical intervention. It is important, however, to consider the proportion of these scans which are returned as normal. Despite this, clinical diagnosis remains difficult, and, at present, more accurate diagnostic or investigative criteria have not been suggested.

Magnetic resonance imaging could not be tolerated by one of our patients because of claustrophobia. In such cases, contrast-enhanced CT of the temporal bones can be used, and ABR may be considered as it can be sensitive in identifying vestibular schwannomas greater than $1 \mathrm{~cm}$ in size. ${ }^{26,27}$

An increasing number of MRI scans are being requested for patients presenting with unilateral audiovestibular symptoms, in order to rule out internal auditory meatus lesions. The majority of these scans report a normal internal auditory meatus, with an incidental finding other than a vestibular schwannoma much 


\begin{tabular}{|c|c|c|c|}
\hline \multicolumn{4}{|c|}{$\begin{array}{c}\text { TABLE I } \\
\text { COMMON INCIDENTAL FINDINGS* }\end{array}$} \\
\hline Incidental finding & $\begin{array}{l}\text { Prevalence } \\
(\%)\end{array}$ & $\begin{array}{l}\text { Most common potential } \\
\text { complications }\end{array}$ & Treatment of asymptomatic findings \\
\hline Arachnoid cyst & 0.5 & $\begin{array}{l}\text { Pressure on adjacent brain } \\
\text { structures }\end{array}$ & $\begin{array}{l}\text { Neurosurgical decompression is not indicated for } \\
\text { asymptomatic cysts (no RCTs) }\end{array}$ \\
\hline Aneurysm & 0.35 & $\begin{array}{l}\text { Haemorrhage (risk } \\
\text { influenced by aneurysm } \\
\text { site \& size) }\end{array}$ & $\begin{array}{l}\text { Endovascular coiling or neurosurgical clipping are } \\
\text { available, but there is uncertainty about their use } \\
\text { because of lack of published RCTs comparing treatment } \\
\text { with conservative management for asymptomatic } \\
\text { aneurysms }\end{array}$ \\
\hline Meningioma & 0.29 & $\begin{array}{l}\text { Pressure on adjacent brain } \\
\text { structures }\end{array}$ & $\begin{array}{l}\text { Neurosurgical excision \& radiotherapy tend to be used } \\
\text { when meningiomas cause symptoms (no RCTs) }\end{array}$ \\
\hline Cavernous malformation & 0.16 & $\begin{array}{l}\text { Haemorrhage, epileptic } \\
\text { seizure }\end{array}$ & $\begin{array}{l}\text { Neurosurgical excision \& stereotactic radiosurgery are } \\
\text { available, but there are no case series or RCTs } \\
\text { supporting their use for asymptomatic cavernous } \\
\text { malformations }\end{array}$ \\
\hline Hydrocephalus & 0.1 & Headache, drowsiness & Shunting is not indicated for people without symptoms \\
\hline $\begin{array}{l}\text { White matter lesions suggestive } \\
\text { of inflammatory disorder }\end{array}$ & 0.06 & $\begin{array}{l}\text { Later development of } \\
\text { multiple sclerosis }\end{array}$ & $\begin{array}{l}\text { Immunological treatments are not indicated. Cautious } \\
\text { medical review \& advice may be needed }\end{array}$ \\
\hline Low grade glioma & 0.05 & $\begin{array}{l}\text { Pressure on adjacent brain } \\
\text { structures, epileptic } \\
\text { seizure }\end{array}$ & $\begin{array}{l}\text { Neurosurgical excision may be used, but who to treat \& } \\
\text { when are uncertain (no RCTs). Occasionally, more } \\
\text { malignant primary brain tumours like glioblastomas } \\
\text { have been reported as first presenting during scanning } \\
\text { for other purposes }\end{array}$ \\
\hline Arteriovenous malformation & 0.05 & $\begin{array}{l}\text { Haemorrhage, epileptic } \\
\text { seizure }\end{array}$ & $\begin{array}{l}\text { Endovascular embolisation, neurosurgical excision \& } \\
\text { stereotactic radiosurgery are available. There is an } \\
\text { ongoing RCT comparing treatment with conservative } \\
\text { management for unruptured arteriovenous } \\
\text { malformations }\end{array}$ \\
\hline $\begin{array}{l}\text { Common developmental } \\
\text { variants, rarely of medical } \\
\text { importance }\end{array}$ & $\begin{array}{l}\text { Precise } \\
\quad \text { unknown }\end{array}$ & & May alarm non-expert \\
\hline
\end{tabular}

*Presented in descending order of their prevalence on brain magnetic resonance imaging, with details of their potential complications and treatment. Reproduced with permission from the Royal College of Radiologists. ${ }^{24} \mathrm{RCT}=$ randomised control trial

more likely to be present. If these findings require no further investigation or management, patients should be reassured, and tinnitus therapy, balance clinic or hearing aids issued if appropriate. ${ }^{28}$

Otorhinolaryngologists should also be mindful that hearing loss could stem from dysfunction anywhere along the auditory pathway (inner ear, vestibulocochlear nerve or higher processing centres of the brain). Therefore, pathology concerning the higher auditory pathways in addition to abnormalities within the internal auditory meatus should not be overlooked, and should be imaged accordingly in patients with unilateral audiovestibular symptoms. Fishman et al. described a few case studies in which the observed pathology (meningioma, demyelinating disease and widespread vasculitis) seemed to have caused the hearing loss, either as a direct effect on the vestibulocochlear nerve or via higher auditory pathways. ${ }^{29}$

In the case of incidental findings requiring intervention, otorhinolaryngologists should have a basic level of understanding of the underlying pathology so that patients can be informed and counselled in clinic, prior to onward referral to the appropriate specialty. Further guidance can be sought by using the 'Management of Incidental Findings Detected during Research Imaging' document produced by the Royal College of Radiologists (Table I). ${ }^{24}$
- Unilateral audiovestibular symptoms are common; internal auditory meatus magnetic resonance imaging (MRI) is used to rule out vestibular schwannomas

- Most internal auditory meatus MRI scans are normal, but incidental findings are not uncommon

- The vestibular schwannoma pick-up rate remains quite low despite various screening criteria

- This cohort study reports on the frequency of incidental findings and their management with reference to literature

- The study aimed to highlight the importance of imaging higher auditory pathways

- It aimed to improve otorhinolaryngologists' knowledge in managing patients with incidental findings and in performing any onward referral

We suggest formulating a local algorithm in conjunction with radiology colleagues for incidental findings, particularly for use in units without easily available specialist neuroradiological, neurological or 
neurosurgical advice, in order to avoid unnecessary concern over the reporting of normal anatomical variants and to facilitate a more efficient referral on to allied specialties.

\section{References}

1 Newton JR, Shakeel M, Flatman S, Beattie C, Ram B. Magnetic resonance imaging screening in acoustic neuroma. $\mathrm{Am} \mathrm{J}$ Otolaryngol 2010;31:217-20

2 Obholzer RJ, Rea PA, Harcourt JP. Magnetic resonance imaging screening for vestibular schwannoma: analysis of published protocols. J Laryngol Otol 2004;118:329-32

3 Dawes PJ, Jeannon JP. Audit of regional screening guidelines for vestibular schwannoma. J Laryngol Otol 1998;112:860-4

4 Selesnick SH, Jackler RK, Pitts LW. The changing clinical presentation of acoustic tumors in the MRI era. Laryngoscope 1993; 103:431-6

5 Sidman JD, Carrasco VN, Whaley RA, Pillsbury HC. Gadolinium: the new gold standard for diagnosing cerebellopontine angle tumors. Arch Otolaryngol Head Neck Surg 1989; 115:1244-7

6 Vandervelde C, Connor SE. Diagnostic yield of MRI for audiovestibular dysfunction using contemporary referral criteria: correlation with presenting symptoms and impact on clinical management. Clin Radiol 2009;64:156-63

7 Papanikolaou V, Khan MH, Keogh IJ. Incidental findings on MRI scans of patients presenting with audiovestibular symptoms. BMC Ear Nose Throat Disord 2010;10:6

8 Katzman GL, Dagher AP, Patronas NJ. Incidental findings on brain magnetic resonance imaging from 1000 asymptomatic volunteers. JAMA 1999;282:36-9

9 Mirza S, Malik TH, Ahmed A, Willatt DJ, Hughes DG. Incidental findings on magnetic resonance imaging screening for cerebellopontine angle tumours. J Laryngol Otol 2006; 114:750-4

10 Robson AK, Leighton SE, Anslow P, Milford CA. MRI as a single screening procedure for acoustic neuroma: a cost effective protocol. J R Soc Med 1993;86:455-7

11 Raber E, Dort JC, Sevick R, Winkelaar R. Asymmetric hearing loss: toward cost-effective diagnosis. J Otolaryngol 1997;26: 88-91

12 Gupta R, Gupta N, Gupta S, Gupta G, Kalsotra P, Sharma R. Incidental findings on magnetic resonance imaging in patients with tinnitus. Indian J Otol 2015;21:41-6

13 Chadha NK, Weiner GM. Vascular loops causing otological symptoms: a systematic review and meta-analysis. Clin Otolaryngol 2008;33:5-11

14 Møller MB, Møller AR, Jannetta PJ, Jho HD. Vascular decompression surgery for severe tinnitus: selection criteria and results. Laryngoscope 1993;103:421-7

15 Tash RR, Kier EL, Chyatte D. Hemifacial spasm caused by a tortuous vertebral artery: MR demonstration. J Comput Assist Tomogr 1988;12:492-4

16 O'Sullivan M, Lythgoe DJ, Pereira AC, Summers PE, Jarosz JM, Williams SC et al. Patterns of cerebral blood flow reduction in patients with ischemic leukoaraiosis. Neurology 2002;59: $321-6$
17 De Leeuw FE, de Groot JC, Achten E, Oudkerk M, Ramos LM, Heijboer R et al. Prevalence of cerebral white matter lesions in elderly people: a population based magnetic resonance imaging study. The Rotterdam Scan Study. J Neurol Neurosurg Psychiatry 2001;70:9-14

18 Debette S, Markus HS. The clinical importance of white matter hyperintensities on brain magnetic resonance imaging: systematic review and meta-analysis. BMJ 2010;341:c3666

19 Moser FG, Panush D, Rubin JS, Honigsberg RM, Sprayregen S, Eisig SB. Incidental paranasal sinus abnormalities on MRI of the brain. Clin Radiol 1991;43:252-4

20 Patel K, Chavda SV, Violaris N, Pahor AL. Incidental paranasal sinus inflammatory changes in a British population. J Laryngol Otol 2007;110:649-51

21 Tarp B, Fiirgaard B, Christensen T, Jensen JJ, Black FT. The prevalence and significance of incidental paranasal sinus abnormalities on MRI. Rhinology 2000;38:33-8

22 Okuda DT, Mowry EM, Beheshtian A, Waubant E, Baranzini $\mathrm{SE}$, Goodin DS et al. Incidental MRI anomalies suggestive of multiple sclerosis: the radiologically isolated syndrome. Neurology 2009;72:800-5

23 Barkhof F, Filippi M, Miller DH, Scheltens P, Campi A, Polman $\mathrm{CH}$ et al. Comparison of MRI criteria at first presentation to predict conversion to clinically definite multiple sclerosis. Brain 1997;120:2059-69

24 Royal College of Radiologists. Management of Incidental Findings Detected during Research Imaging. London: Royal College of Radiologists, 2011

25 Cueva RA. Auditory brainstem response versus magnetic resonance imaging for the evaluation of asymmetric sensorineural hearing loss. Laryngoscope 2004;114:1686-92

26 Stachler RJ, Chandrasekhar SS, Archer SM, Rosenfeld RM, Schwartz SR, Barrs DM et al. Clinical practice guideline: sudden hearing loss. Otolaryngol Head Neck Surg 2012;146(3 suppl):S1-35

27 Fortnum H, O'Neill C, Taylor R, Lenthall R, Nikolopoulos T, Lightfoot $\mathrm{G}$ et al. The role of magnetic resonance imaging in the identification of suspected acoustic neuroma: a systematic review of clinical and cost effectiveness and natural history. Health Technol Assess 2009;13:1-154

28 Reeves D, Mason L, Prosser H, Kiernan C. Direct Referral Systems for Hearing Aid Provision. London: HMSO, 1994

29 Fishman JM, Shoeb M, Abramovich S. Importance of imaging the central auditory pathways in cases of unilateral sensorineural hearing loss. J Laryngol Otol 2008;122:1-2

Address for correspondence:

Ms Hay Mar Htun,

Blackpool Victoria Hospital,

Whinney Heys Road,

Blackpool FY3 8NR, UK

E-mail: haymarhtun@doctors.org.uk

Ms H M Htun takes responsibility for the integrity of the content of the paper

Competing interests: None declared 